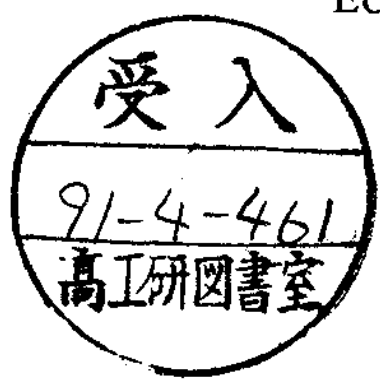

CERN/PPE 91-51

PPE-LAA-SF 91-5

7 March 1991

\title{
REFLECTION LOSSES IN POLYSTYRENE FIBRES $\left.{ }^{*}\right)$
}

C. D'Ambrosio, H. Leutz and M. Taufer

CERN, Geneva, Switzerland

T. Shimizu and O. Shinji

Kuraray Co. Ltd., Tokyo, Japan Turatay, $4=17$,

J. Sun

World Laboratory, Lausanne, Switzerland

\begin{abstract}
The reflection losses, which occur during light guidance through polystyrene fibres of $30 \mu \mathrm{m}$ diameter at their core-cladding interface, have been determined in order to evaluate their contribution to the total light attenuation of scintillating fibres. For the deviation $(1-q)$ of the reflection coefficient $q$ from unity we obtained: $6 \times 10^{-5}$ (at $458 \mathrm{~nm}$ ), $5.36 \times 10^{-5}$ (at $514 \mathrm{~nm}$ ) and $5 \times 10^{-5}$ (at $633 \mathrm{~nm}$ ). In addition, we found light losses in the polystyrene core, which we attribute to Rayleigh scattering on submicron density variations in polystyrene. The resulting scattering lengths are: $3.6 \mathrm{~m}$ (at $420 \mathrm{~nm}$ ) and $9.5 \mathrm{~m}$ (at $530 \mathrm{~nm}$ ).
\end{abstract}

Submitted to Nuclear Instruments and Methods

(*) The work reported here is part of the LAA project.

$\mathrm{Mac} / \mathrm{PPE} / 6004 \mathrm{P} / \mathrm{HL} / \mathrm{sj}$ 


\section{INTRODUCTION}

Light attenuation in scintillating fibres is caused partly by bulk attenuation in the fibre core and partly by reflection losses of the guided light emission at the surrounding corecladding interface. Whereas the bulk attenuation in the fibre core results mainly from the long-wavelength tail of the dopant's absorption band, the reflection losses are due to different kinds of imperfections at the core-cladding interface. These losses occur more or less at each total light reflection, which results from the difference between the corresponding refraction indices $\left(n_{\text {core }}>n_{\text {clad }}\right)$. The integral reflection loss is therefore directly proportional to the number of reflections, which in turn is inversely proportional to the fibre diameter.

The proportion between the two corresponding sources of light losses therefore depends mainly on the fibre diameter. Whereas for larger fibre diameters $(>0.5 \mathrm{~mm})$ the contribution of reflection losses is small compared to the bulk attenuation, they become increasingly important for smaller diameters $(<0.1 \mathrm{~mm})$. Consequently, it is essential to manufacture small diameter scintillating fibres with high-quality core-cladding interfaces. To quantitatively evaluate these reflection losses, it is necessary to separate them from the bulk attenuation. To our knowledge, such a direct measurement of reflection losses has never been performed before. For this purpose, we developed a measuring procedure to determine reflection losses at the core-cladding interface independently from the bulk attenuation in the fibre core [1-4]. This article reports on such measurements performed with polystyrene fibres ${ }^{(*)}$ of $0.03 \mathrm{~mm}$ diameter at $633 \mathrm{~nm}, 514 \mathrm{~nm}$ and $458 \mathrm{~nm}$ wavelengths, which do not contain any dopant.

\section{RELATIONS FOR LIGHT GUIDANCE THROUGH FIBRES}

The incident light intensity $\mathrm{I}_{0}$ and the intensity $\mathrm{I}$, transmitted after $\mathrm{N}$ reflections at the core-cladding interface are related by

$$
\mathrm{I}=\mathrm{I}_{0} \mathrm{q}^{\mathrm{N}}
$$

where $\mathrm{q} \leq 1$ means the reflection coefficient, the value of which we want to determine.

A meridional $\left(^{(*)}\right)$ light ray injected at the centre of the fibre core (refractive index $n_{1}$ ) and being subsequently reflected at the cladding (refractive index $n_{2}<n_{1}$ ) suffers

$$
\mathrm{N}=\frac{\mathrm{L}}{\mathrm{d}} \operatorname{tg} \vartheta
$$

reflections. $\mathrm{L}$ and $\mathrm{d}$ mean fibre length and diameter, resp.; $\vartheta$ is the supplement of the reflection angle $\alpha$ at the core-cladding interface (fig. 1).

$\left({ }^{*}\right)$ Manufactured by Kuraray Co., Ltd, Tokyo, Japan.

(**) Skew light rays, as partly produced by ionizing radiation traversing scintillating fibres, are mostly diffused outside the fibre core, after short travelling distances. 
Relations (1) and (2) can be combined to

$$
\ln \left(\frac{\mathrm{I}}{\mathrm{I}_{0}}\right)=-(1-\mathrm{q}) \frac{\mathrm{L}}{\mathrm{d}} \operatorname{tg} \vartheta
$$

if we express $\ell \mathrm{n} \mathrm{q}$ by $(1-\mathrm{q})$, which is permitted, since $\mathrm{q}$ differs always less than $1 \%$ from unity. To extend relation (3) to all light rays, we integrate over the solid angle subtended by the injected light cone with a numerical aperture $N A_{i}=\sin \theta_{i}=n_{1} \sin \vartheta_{i}$ (fig. 1)

$$
\int_{0}^{\theta_{\mathrm{i}}} \frac{\mathrm{I}}{\mathrm{I}_{0}} \mathrm{dI}=\int_{0}^{\theta_{\mathrm{i}}} \mathrm{e}^{-(1-q) \frac{\mathrm{L}}{\mathrm{d}} \mathrm{t} \vartheta} \sin \vartheta \mathrm{d} \vartheta\left(\int_{0}^{\theta_{\mathrm{i}}} \sin \vartheta \mathrm{d} \vartheta\right)^{-1},
$$

where the integral in the denominator provides the normalization to unity for zero fibre length $\mathrm{L}$.

If the numerical aperture $N A_{i}$ of the injected light exceeds that of the fibre $\left(N A_{f}=n_{1}\right.$ $\sin \vartheta_{c}$ ), all light rays with angles larger than the critical angle $\vartheta_{c}$ of the total reflection escape from the fibre. This case changes relation (4) to

$$
\int_{0}^{\vartheta_{i}} \frac{\mathrm{I}}{\mathrm{I}_{0}} \mathrm{dI}=\left(1-\cos \vartheta_{c}\right) \int_{0}^{\vartheta_{c}} \mathrm{e}^{-(1-q) \frac{\mathrm{L}}{\mathrm{d}} \mathrm{tg} \vartheta} \sin \vartheta \mathrm{d} \vartheta\left(\left(1-\cos \vartheta_{\mathrm{i}}\right) \int_{0}^{\vartheta_{c}} \sin \vartheta \mathrm{d} \vartheta\right)^{-1}
$$

The term $\left(1-\cos \vartheta_{c}\right)$ represents the amount of light trapped by the fibre, which must be divided by the larger amount of the injected light $\left(1-\cos \vartheta_{i}\right)$ to obtain the trapped fraction. The integral, limited by the critical angle $\vartheta_{\mathrm{c}}$ is constant.

As a useful approximation, which is correct if

$$
\int_{0}^{\vartheta_{i}} \frac{\mathrm{I}}{\mathrm{I}_{0}} \mathrm{dI} \geq 1 / \mathrm{e}
$$

i.e. within the first reflection length $\Lambda_{\mathrm{R}}$, we can replace relation (4) by

$$
\ln \int_{0}^{\vartheta_{\mathrm{i}}} \frac{\mathrm{I}}{\mathrm{I}_{0}} \mathrm{dI}=-(1-\mathrm{q}) \frac{\mathrm{L}}{\mathrm{d}} \int_{0}^{\vartheta_{\mathrm{i}}} \vartheta^{2} \mathrm{~d} \vartheta\left(\int_{0}^{\vartheta_{\mathrm{i}}} \vartheta \mathrm{d} \vartheta\right)^{-1}=-(1-\mathrm{q}) \frac{\mathrm{L}}{\mathrm{d}} \frac{2}{3} \vartheta_{\mathrm{i}}
$$

which yields for $\vartheta_{\mathrm{i}} \approx \mathrm{NA}_{\mathrm{i}} / \mathrm{n}_{1}$

$$
\int_{0}^{\vartheta_{i}} \frac{\mathrm{I}}{\mathrm{I}_{0}} \mathrm{dI}=\mathrm{e}^{-\frac{1-\mathrm{q}}{1.5} \frac{\mathrm{L}}{\mathrm{d}} \frac{N A_{\mathrm{i}}}{\mathrm{n}_{1}}}
$$

and

$$
\Lambda_{\mathrm{R}}=\frac{1.5}{1-\mathrm{q}} \frac{\mathrm{n}_{1}}{\mathrm{NA}_{\mathrm{f}}} \mathrm{d}
$$


if we take $L=\Lambda_{R}$ for

$$
\int_{0}^{\theta_{i}} \frac{I}{I_{0}} d I=\frac{1}{e} \text {. }
$$

$\Lambda_{R}$ therefore means the fibre length, after which the initial light intensity $I_{0}$ is reduced to $1 / \mathrm{e}$ due to reflection losses.

\section{EXPERIMENTAL PROCEDURE}

To determine the value $(1-q)$ according to relations (4) and (5), we must avoid any absorption in the fibre core. Therefore, we investigated undoped fibres in order not to be affected by the absorption band of the scintillator. In this way we measured the reflection losses at $458 \mathrm{~nm}, 514 \mathrm{~nm}$ and $633 \mathrm{~nm}$ wavelengths.

For any given fibre production, the diameter $d$ and the refractive index $n_{1}$ of the fibre core are fixed values. We are left with the fibre length $L$ and the angle $\vartheta$ (relations (4) and (5)) as free parameters. The best way for changing the angle $\vartheta$ (fig. 1 ), is via the numerical aperture $\mathrm{NA}_{\mathrm{i}}$ of the injected light cone

$$
\vartheta=\arcsin \left(\frac{N A_{i}}{n_{1}}\right) .
$$

We applied an experimental layout as presented in fig. 2. The light source is either a He-Ne laser, which emits at $632.8 \mathrm{~nm}$ or, alternatively, an Ar laser tuned for $514 \mathrm{~nm}$ or $458 \mathrm{~nm}$ emissions. The numerical aperture $\mathrm{NA}_{\mathbf{i}}$ of the incident light $\mathrm{I}_{0}$ can be varied between 0.1 and 0.85 by appropriate setting of lens 1 with respect to the two microscope objectives $M_{1}$, which follow the beam splitter $\mathrm{BS}_{1}$. Incident $\left(\mathrm{I}_{0}\right)$ and transmitted (I) light intensities are compared via the two beam splitters $\left(\mathrm{BS}_{1}, \mathrm{BS}_{2}\right)$ and the two photodiodes $\left(\mathrm{Ph}_{1}, \mathrm{Ph}_{2}\right)$ by the indicated ratiometer. Corresponding tests affirmed that intensity variations of $\mathrm{I}_{0}$ did not affect the $\mathrm{I} / \mathrm{I}_{0}$ ratio.

The fibres were produced as fused bundles of square cross sections with $1 \mathrm{~mm}$ edge length. Such a bundle contains around 900 individual hexagonal fibres of $30 \mu \mathrm{m}$ core diameter, separated from each other by a $5 \mu \mathrm{m}$ cladding as displayed in fig. 3 . For the measurement of the intensity ratios

$$
\int_{0}^{\vartheta_{i}} \frac{I}{I_{0}} d I,
$$

the incident light cone was focused via $\mathrm{M}_{1}$ on the centre of an individual fibre. The focus position could be verified on the display screen of the CCD camera (fig. 2), which received half the transmitted light through the beam splitter $\mathrm{BS}_{2}$. The correct focus position was achieved, if the camera screen displayed a uniformly illuminated fibre, surrounded by its six neighbours, all at equal but about three orders of magnitude lower light level than the central 
fibre (fig. 4). The measured focal size at the fibre entrance face was of $\approx 10 \mu \mathrm{m}$ diameter. Therefore, we kept the injected light power below $50 \mu \mathrm{W}$ to avoid overpowering of the fibre end at the focal point. In spite of the hexagonal fibre shape, our assumption of injecting mainly meridional rays is justified with this arrangement.

To distinguish between the light intensity directly transmitted through the illuminated fibre and the total light intensity emitted from the end face of the fibre bundle, we could optionally insert a pinhole (fig. 2), which stops all diffused light carried by other fibres of the bundle. The intensity difference between the non-pinhole and the pinhole set-up yielded finally the light intensity diffused from the illuminated fibre into other fibres of the bundle.

\section{RESULTS AND DISCUSSION}

The integrated intensity ratios of incident $\left(\mathrm{I}_{0}\right)$ and transmitted light $(\mathrm{I})$ are plotted in fig. 5 for different fibre lengths $\mathrm{L}$ and for $458 \mathrm{~nm}$ wavelength of the incident light versus the injected numerical aperture $\mathrm{NA}_{i}$, which is related to the reflection angle $\vartheta$ (fig. 1) according to equation (7). The short fibre sample of $15 \mathrm{~mm}$ length was measured to normalize the intensity ratios to unity for zero aperture and zero fibre length. The indicated curves were fitted according to relation (4) $\left(0 \leq \mathrm{NA}_{\mathrm{i}} \leq \mathrm{NA}_{\mathrm{f}}\right)$ and relation (5) $\left(\mathrm{NA}_{\mathrm{f}}<\mathrm{NA}_{\mathrm{i}} \leq 1\right)$. The transition from $\mathrm{NA}_{\mathrm{i}} \leq \mathrm{NA}_{\mathrm{f}}$ to injected numerical apertures larger than that of the fibre $\left(\mathrm{NA}_{\mathrm{f}}\right)$ can be clearly recognized for the shorter fibres. The integrated intensity ratios for the $10 \mathrm{~m}$ long fibre are displayed in fig. 6 for $458 \mathrm{~nm}$ (blue), $514 \mathrm{~nm}$ (green) and $633 \mathrm{~nm}$ (red) wavelengths. Again, these ratios are normalized to unity for zero aperture and fibre length. The curves are fitted to the measured values according to relations (4) and (5).

Taking the values measured for $\mathrm{NA}_{\mathrm{i}}<\mathrm{NA}_{\mathrm{f}}$, we calculated the $(1-\mathrm{q})$ - values from relation (4). These values are plotted in fig. 7 for the three wavelengths. The least squares fits yield the following results:

$$
\begin{array}{ll}
458 \mathrm{~nm} \text { (blue) : } & 1-\mathrm{q}=(5.98 \pm 0.10) \times 10^{-5}, \\
514 \mathrm{~nm}(\text { green }): & 1-\mathrm{q}=(5.36 \pm 0.08) \times 10^{-5}, \\
633 \mathrm{~nm}(\text { red }): & 1-\mathrm{q}=(5.00 \pm 0.05) \times 10^{-5}
\end{array}
$$

These values contrast clearly with those estimated by Davis et al. [5], who find not only (1 q) values much higher than ours, but also a strong wavelength dependence of a factor 2.8 between $450 \mathrm{~nm}$ and $480 \mathrm{~nm}$.

From fig. 6 we learn that the integrated intensity ratios increase with the wavelength of the injected light. This is already true for the intercepts of the fitted curves to zero numerical aperture $\mathrm{NA}_{\mathrm{i}}$. This means that the intensity losses occur already for light travelling through the fibre core parallel to its axis without touching the core-cladding interface. In this case, the 
injected light does not suffer from reflection losses anymore and its attenuation follows the relation

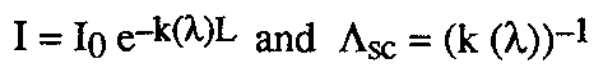

with $\mathrm{k}$ meaning the attenuation coefficient, which depends on the wavelength $\lambda$. This coefficient is plotted in fig. 8(a) for the three intercepts of the $10 \mathrm{~m}$ fibres (fig. 6), versus $\lambda^{-4}$. Since the integrated intensity ratios are normalized to unity via the short fibre samples, we indicated the zero origin of the plot with its error margin as an additional value. The obvious $\lambda^{-4}$ dependence appearing in fig. 8(a) shows that the bulk attenuation in the fibre core is caused by Rayleigh scattering. In order to confirm this assumption, we measured carefully the transmission of a $10 \mathrm{~cm}$ long pure polystyrene bulk sample. After deduction of the reflection losses at the entrance and exit face of the sample, the remaining attenuation coefficient versus $\lambda^{-4}$ between $400 \mathrm{~nm}$ and $800 \mathrm{~nm}$ is displayed in fig. $8(\mathrm{~b})$. The absorption peaks overlayed to an again $\lambda^{-4}$ dependence are the harmonics of the CHbonding absorptions at $3.25 \mu \mathrm{m}$ and $3.49 \mu \mathrm{m}$ wavelength, which are beyond the range of our plot. The two weak negative peaks are caused by light emissions of small impurities in the polystyrene bulk.

These Rayleigh scatterings must occur on submicron centres like density variations within the polystyrene. Comparison between the attenuation coefficients in figs 8 (a) and 8(b) shows that the bulk sample scatters around 2.5 times more than the fibre core. Obviously, these scattering losses depend on the production procedure which influences the arrangement and length of the polystyrene molecular chains. From the values of the scattering coefficients we evaluate for $420 \mathrm{~nm}$ wavelength, which corresponds to the emission maxima of most scintillators, a $3.6 \mathrm{~m}$ scattering length $\Lambda_{\mathrm{sc}}$ according to relation (8). This increases to $\Lambda_{\mathrm{Sc}}=9.5 \mathrm{~m}$ at $530 \mathrm{~nm}$, which corresponds to the $3-\mathrm{HF}$ emission maximum.

The $(1-q)$ values also show a wavelength dependence. They are plotted with zero suppression again versus $\lambda^{-4}$ in fig $8(\mathrm{c})$. This shows that the reflection losses are mainly caused by macroscopic defects at the core-cladding interface, overlayed by a small contribution of Rayleigh scattering. At infinite wavelength we obtain, according to relation (6a) a reflection length $\Lambda_{R}(\infty)$ of $2.81 \mathrm{~m}$. This decreases to $2.0 \mathrm{~m}$ reflection length $\Lambda_{\mathrm{R}}$ (420) at $420 \mathrm{~nm}$ wavelength. The difference is due to the Rayleigh scattering contribution, which amounts to $\Lambda_{\mathrm{R}}$ (scatter) $=6.94 \mathrm{~m}$.

After removing the pinhole from the set-up of fig. 2, we measured also the integrated intensity ratios of the whole fibre bundle. Subtraction of the corresponding ratios of the illuminated fibre (pinhole inserted) yields the light fraction, which was diffused from this fibre into the 900 other fibres of the fused bundle. The diffused light fraction

$$
\left(\mathrm{I}_{\mathrm{b}}-\mathrm{I}_{\mathrm{f}}\right) / \mathrm{I}_{\mathrm{f}}=\text { noise/signal }
$$


is plotted versus the numerical aperture of the incident light in fig. 9(a), where $\mathrm{I}_{\mathrm{b}}$ means the total light emitted from all fibres of the multibundle (pinhole not inserted), and $\mathrm{I}_{\mathrm{f}}$ the light intensity (signal) emitted from the illuminated fibre only (pinhole inserted).

For an approximative simulation we write the noise-to-signal ratio of relation (9)

$$
\left(\mathrm{I}_{\mathrm{b}}-\mathrm{I}_{\mathrm{f}}\right) / \mathrm{I}_{\mathrm{f}} \approx \delta\left(1-\mathrm{I}_{\mathrm{f}}\right) \mathrm{e}^{-\mathrm{k}^{\mathrm{T}} \mathrm{L}}+\alpha(\mathrm{L})
$$

with $\delta \approx 0.15$ meaning the fraction of escaping light guided by all the other fibres of the multibundle and $\alpha$ the fraction of light diffused close to the entrance face of the bundle, which goes to zero after a few centimetres. From fig. $9(\mathrm{a})$, we estimate $\alpha(0) \approx 0.04$; and $\mathrm{k}^{\prime}\left(=0.13 \mathrm{~m}^{-1}\right)$ takes into account the attenuation of the diffused light which is composed of reflection losses and scattering within the fibre cores. Finally, If is taken from relations (4) and (5), depending on the value $\mathrm{NA}_{\mathbf{i}}$ of the numerical aperture for the incident light cone. The simulated noise-to-signal ratios are displayed in fig. $9(\mathrm{~b})$ for different fibre lengths. It should be stressed that the ratios of figs 9(a) and 9(b) correspond to the noise of all 900 fibres in the multibundle but to the signal of one illuminated fibre only.

In conclusion, the deviation of the total reflection coefficient $\mathrm{q}$ from unity, amounts to between $5 \times 10^{-5}$ and $6.5 \times 10^{-5}$ for $633 \mathrm{~nm}$ and $420 \mathrm{~nm}$, respectively. According to relation (6a) this results in a reflection length $\Lambda_{R}$ of $2.0 \mathrm{~m}$ for $30 \mu \mathrm{m}$ fibres at $420 \mathrm{~nm}$, which is composed of $\Lambda_{R}(\infty)=2.8 \mathrm{~m}$ for macroscopic defects, and of $\Lambda_{R}$ (scatter) $=7.0 \mathrm{~m}$ caused by Rayleigh losses at the core-cladding interface. It should be stated that all attenuation lengths given in this article are defined as the fibre length, after which the light intensify $I_{0}$ (at zero length) drops to $1 / \mathrm{e}$ due to the light loss in question. This definition contrasts to most indications from other authors, which are based on linear fits to measured intensity attenuations starting at different fibre lengths only, but never at zero.

\section{Acknowledgements}

We wish to thank Professor A. Zichichi for his continuous interest and support for our work. We also acknowledge substantial help from W. Seidl and A. Bochaton. 


\section{REFERENCES}

[1] M. Born and E. Wolf, Principles of optics, Pergemon Press, sixth corrected edition (1986).

[2] S. Solimeno, B. Crosignani and P. Di Porto, Guiding, diffraction and confinement of optical radiation, Academic Press (1986).

[3] D. Marcuse, Principles of optical fibres measurements, Academic Press (1986).

[4] CSELT, Optical fibre communication, Mc Graw-Hill Book company (1981).

[5] A.J. Davis et al., Scintillating optical fibre trajectory detectors, Nucl. Instr. and Meth., A276 (1989) 347-358. 


\section{FIGURE CAPTIONS}

Fig. 1 Light cone injected with a numerical aperture $\mathrm{NA}_{\mathrm{i}}$, and passing through a stepindex fibre under the total reflection angle $\alpha$.

Fig. 2 Experimental lay-out for the measurements.

Fig. 3 Fused fibre bundle of $1 \mathrm{~mm}$ edge length. It contains around 900 individual fibres of hexagonal shape ( $30 \mu \mathrm{m}$ diameter), separated from each other by $5 \mu \mathrm{m}$ PMMA cladding.

Fig. 4 The illuminated fibre ( $30 \mu \mathrm{m}$ diameter) surrounded by its six closest neighbours photographed by the CCD camera. The overexposed central fibre emits about three orders of magnitude more light than its neighbour fibres.

Fig. 5 Light ratios obtained for different fibre lengths at $633 \mathrm{~nm}$ wavelength. They are normalized to unity for zero fibre length and light injected parallel to the fibre axis $\left(\mathrm{NA}_{\mathrm{i}}=0\right)$. The curves are fitted to the measured values according to relations (4) and (5). The indicated errors are due to the normalization of the intensity ratios and to the calibration of the numerical apertures $\mathrm{NA}_{\mathrm{i}}$ of the injected light.

Fig. 6 Light ratios measured with the $10 \mathrm{~m}$ long fibre at $458 \mathrm{~nm}$ (blue), $514 \mathrm{~nm}$ (green), and $633 \mathrm{~nm}$ (red) wavelength. These ratios are also normalized to unity for zero fibre length and aperture $\mathrm{NA}_{\mathrm{i}}$. Curves are fitted to the measured values according to relations (4) and (5). Error sources are the same as in fig. 5.

Fig. $7(1-q)$ values obtained from the light ratios of the $10 \mathrm{~m}$ fibre by applying relation (4). The averaged values are indicated for each colour.

Fig. 8 (a) Attenuation coefficients of $10 \mathrm{~m}$ polystyrene fibres for parallel injected light $\left(\mathrm{NA}_{\mathrm{i}}=0\right)$, i.e. no reflections at the core-cladding interface. The intensity ratios, which yield these coefficients, are again normalized to unity for zero fibre length. The error sources are the same as in fig. 5 .

(b) Attenuation coefficients of a $10 \mathrm{~cm}$ long polystyrene bulk sample. The absorption peaks are due to the harmonics of the $\mathrm{CH}$ bondings with principal absorptions at $3.25 \mu \mathrm{m}$ and $3.49 \mu \mathrm{m}$. The dashed line indicates the exact $\lambda-4$ dependence.

(c) Wavelength dependence of the measured $(1-q)$ values. Note the zero suppression of the $(1-q)$ axis. At $420 \mathrm{~nm}$ the Rayleigh scattering contributes with $\sim 30 \%$. 


\section{FIGURE CAPTIONS (Cont'd)}

Fig. 9 (a) Noise-to-signal ratios at $633 \mathrm{~nm}$ for different fibre lengths, derived from the differences between intensity ratios obtained without and with pinhole inserted into the arrangement of fig. 2 . Because of subtractions between nearly equal values, these ratios are affected with large error margins.

(b) Simulations of the noise-to-signal ratios according to relation (10). They show the same behaviour for the different fibre lengths as the measured ratios in fig. 9(a). 


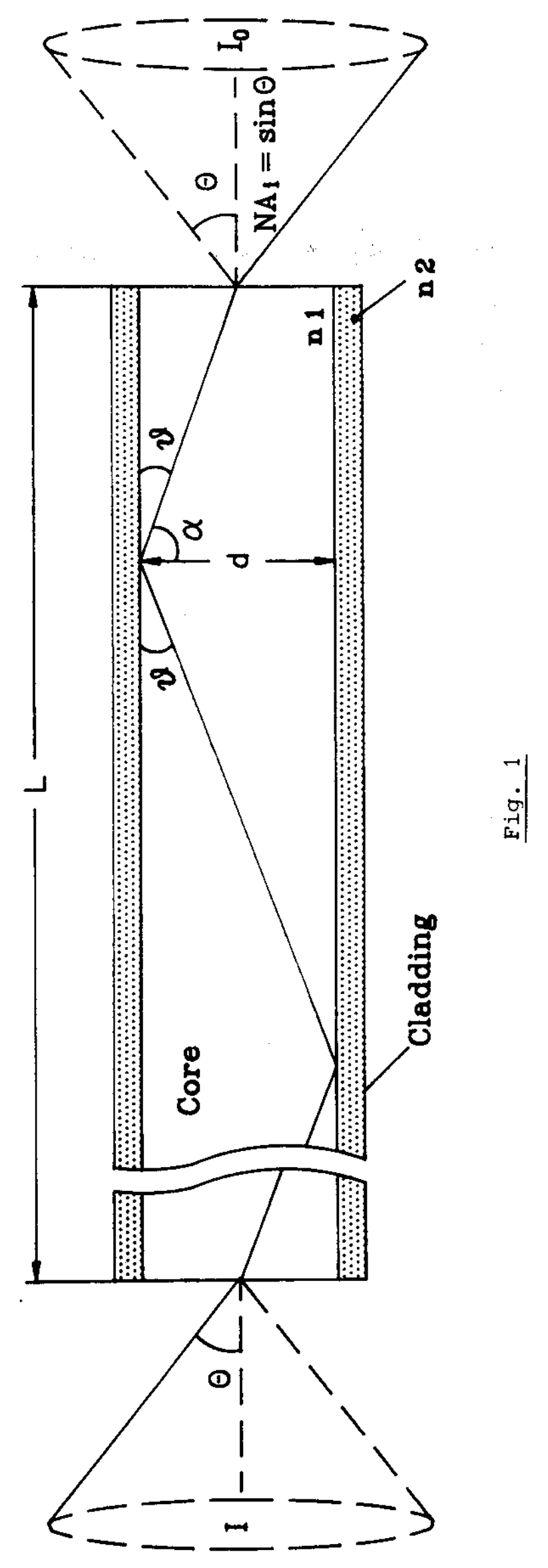




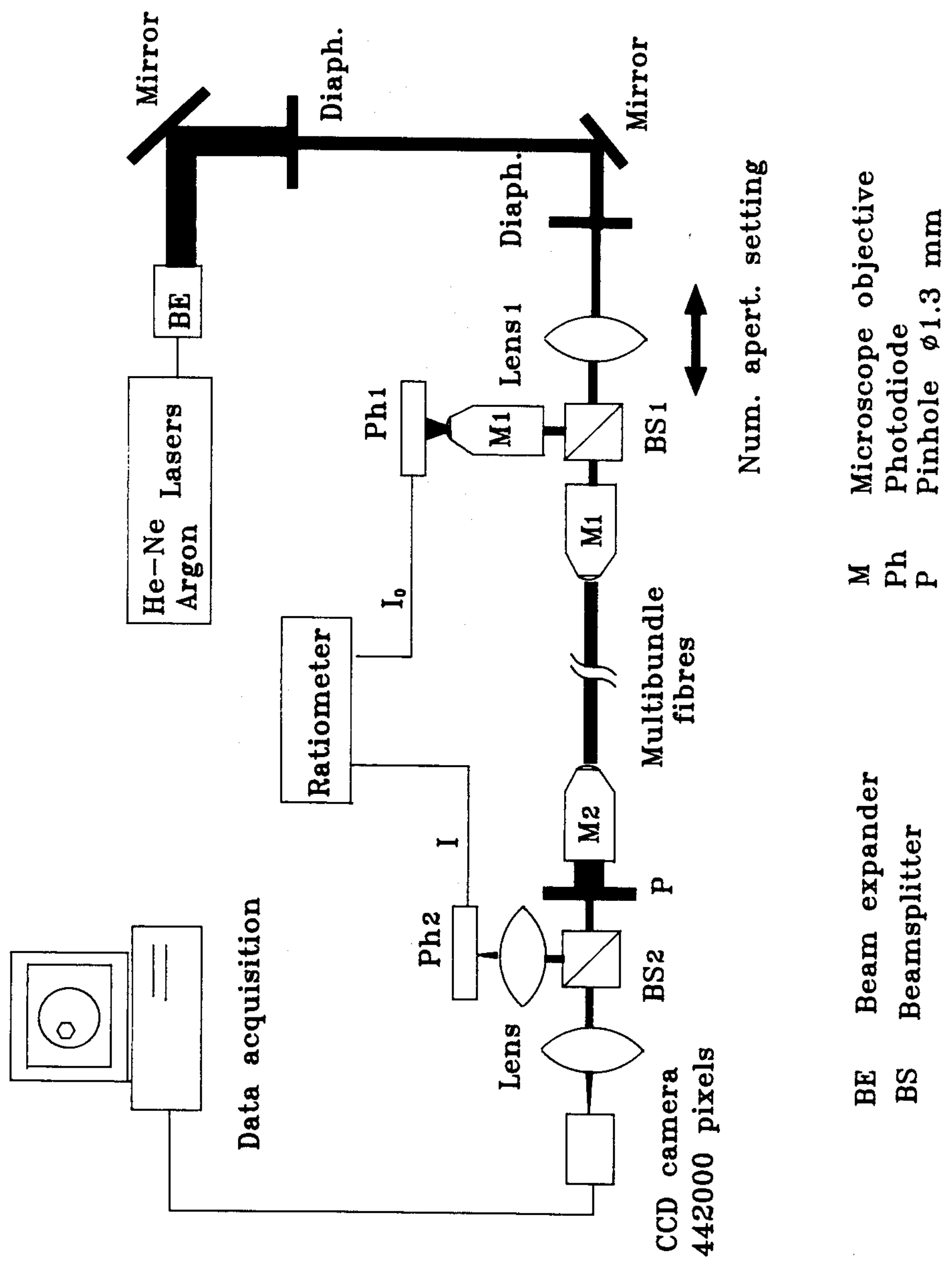




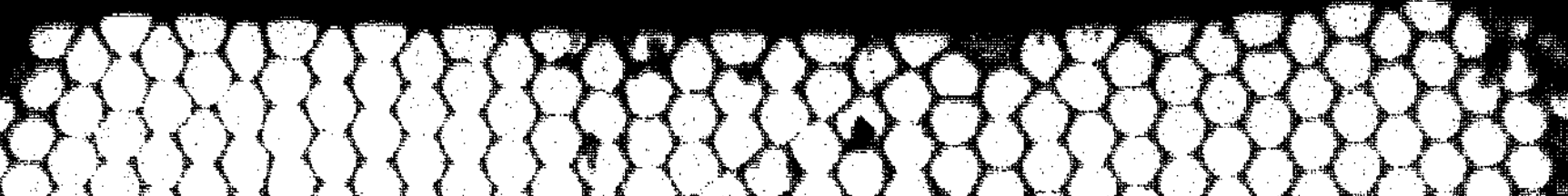

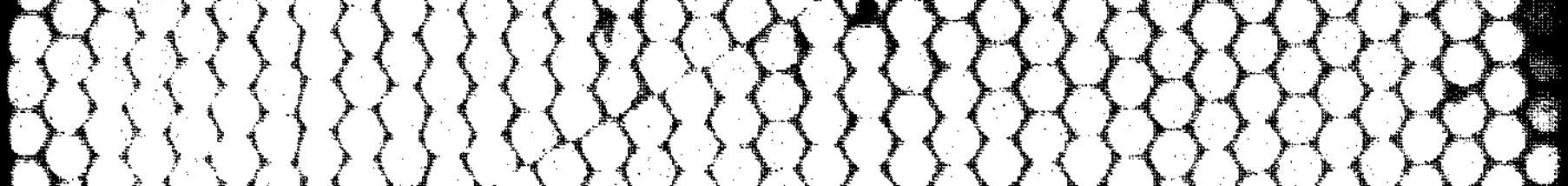
c. 03030303030303030030

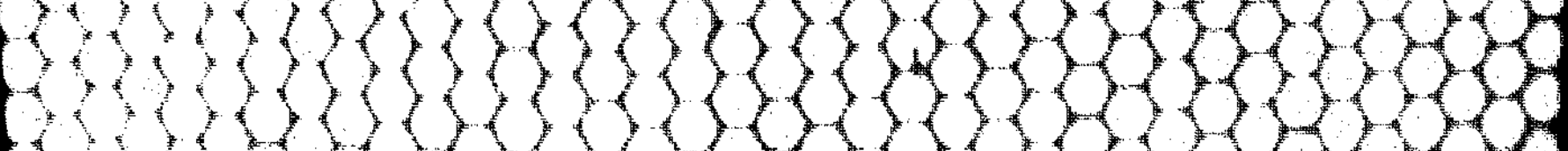

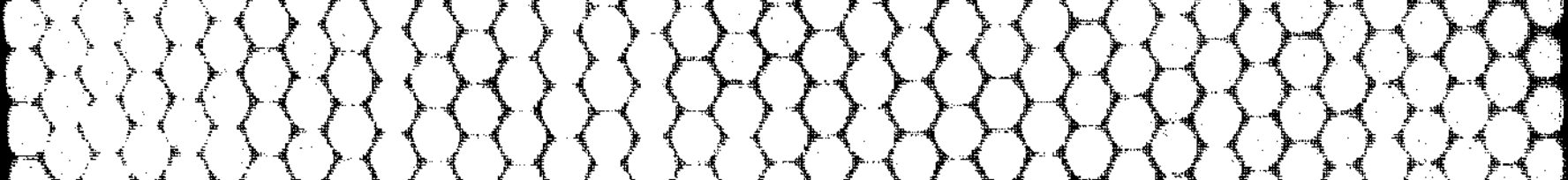

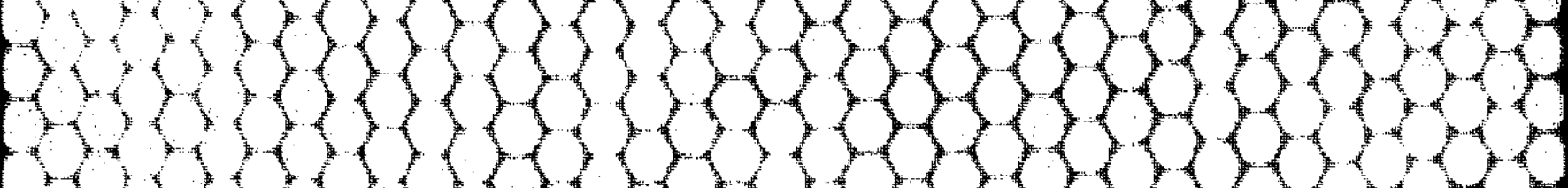
m.

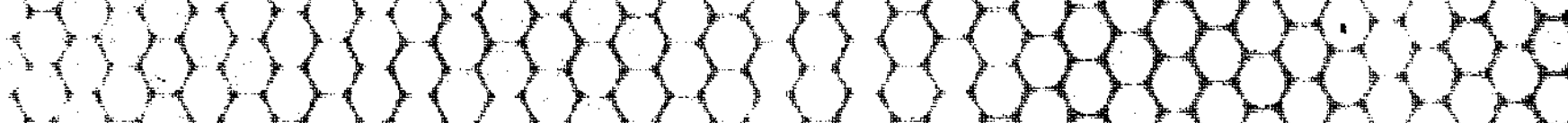
D.

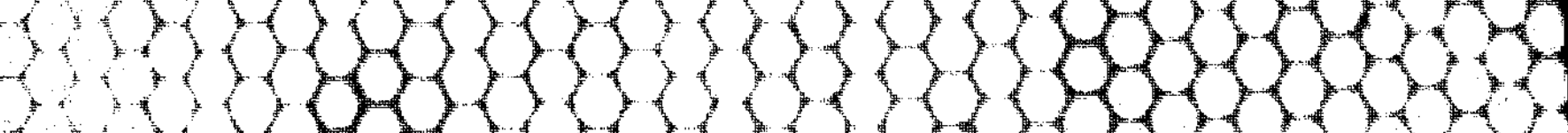

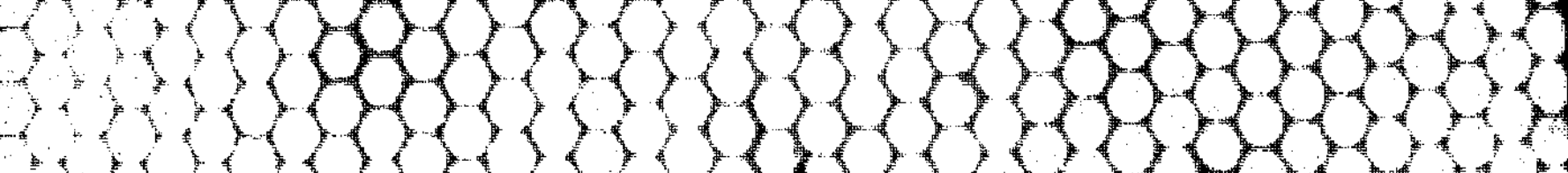

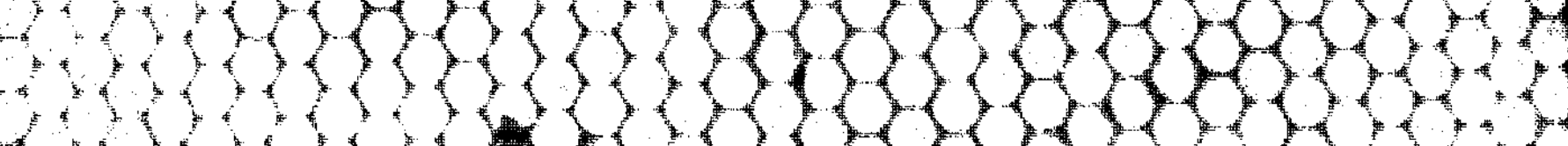
6.

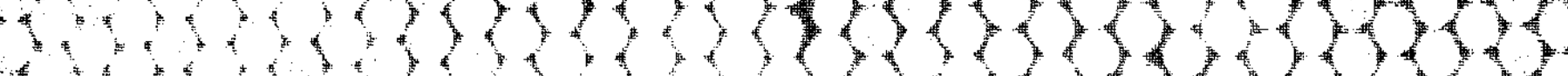
F.

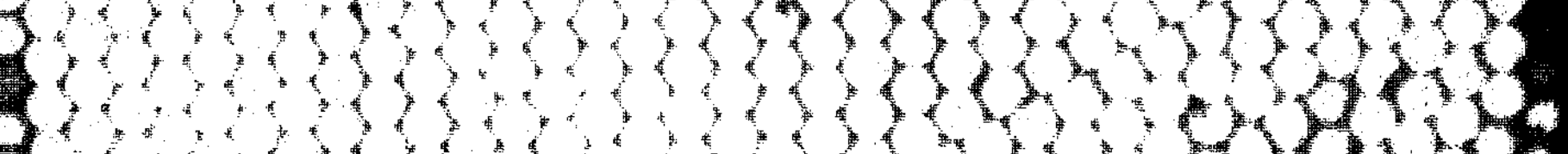

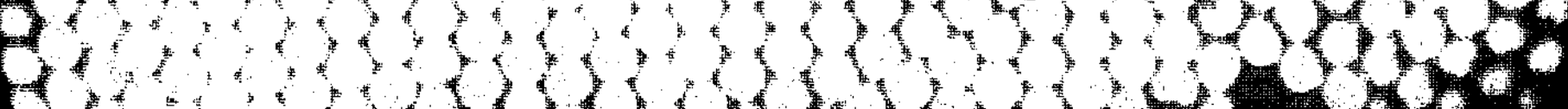

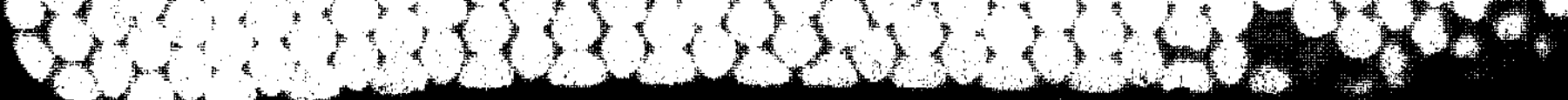




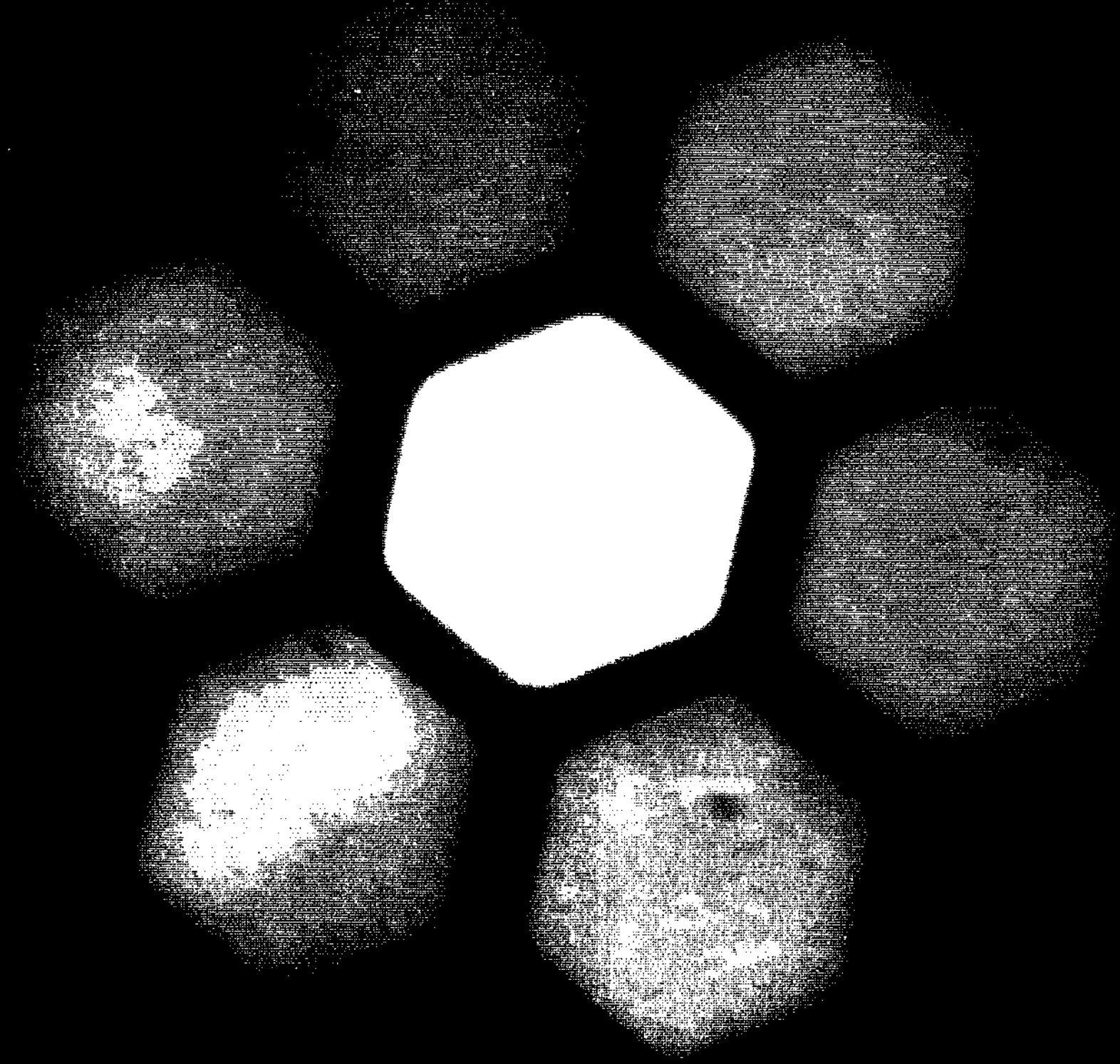

Fig. 4 


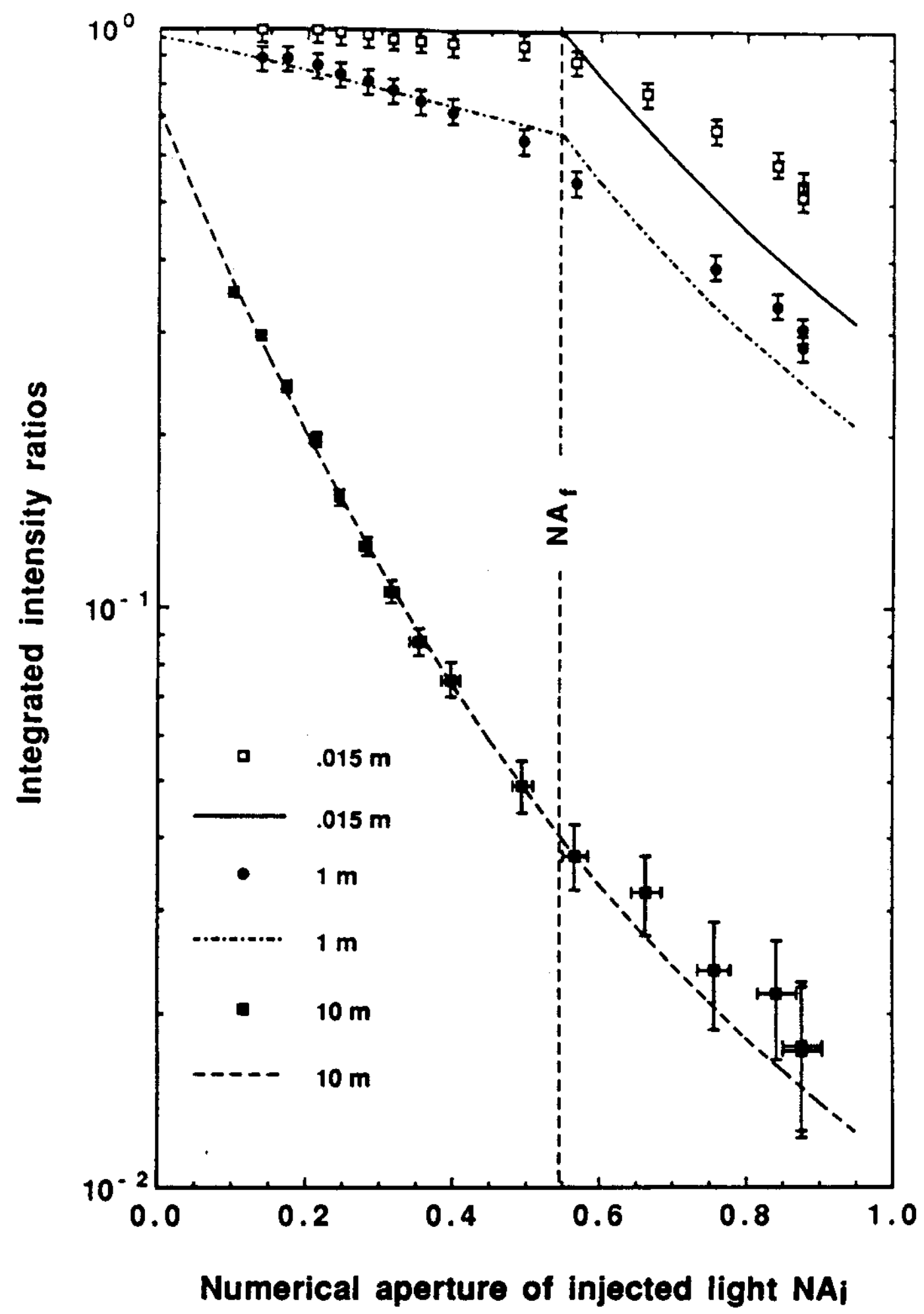

Fig. 5 


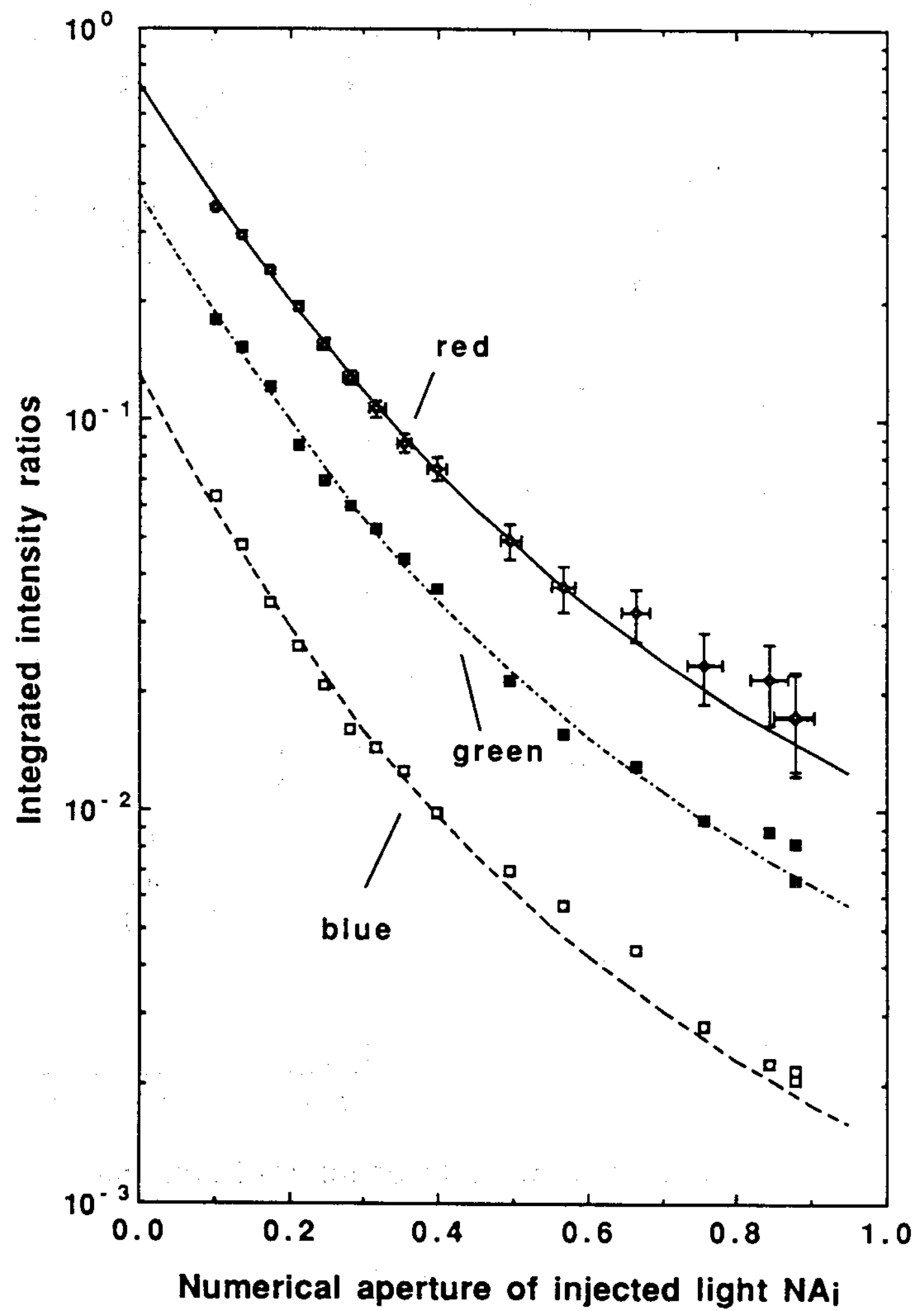

Fig. 6 

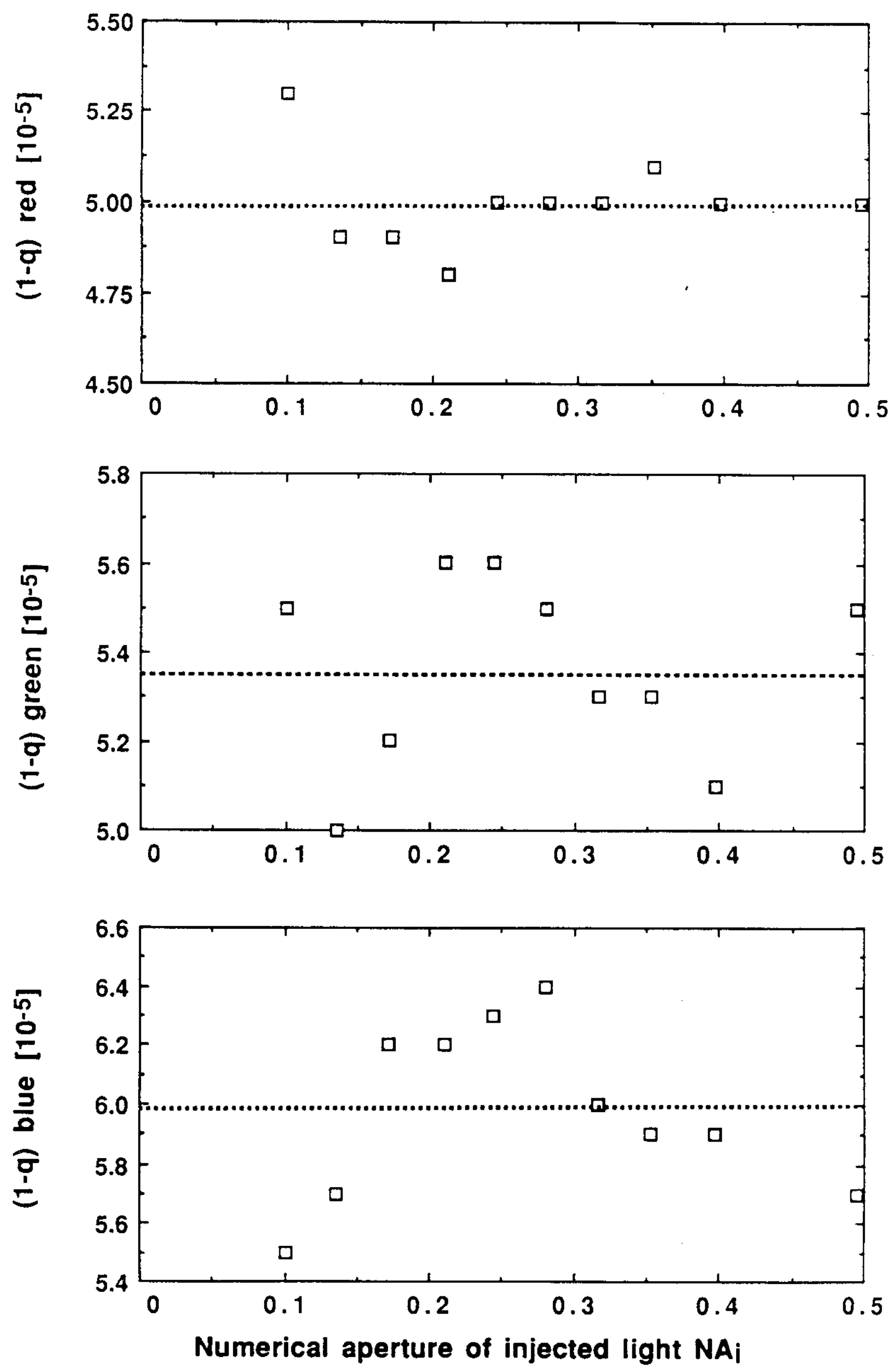

Fig. 7 


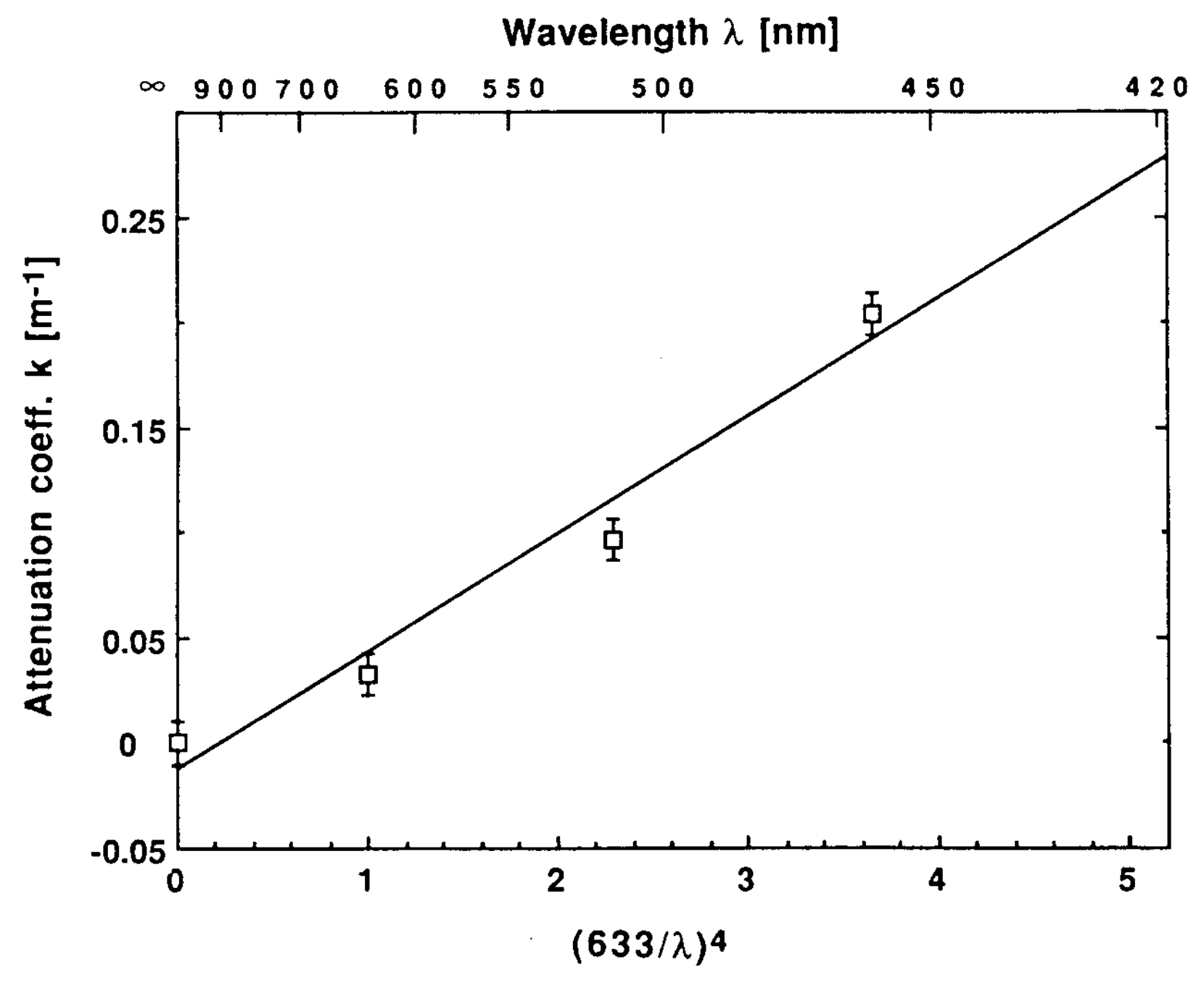

Fig. 8(a) 
Wavelength $\lambda$ [nm]

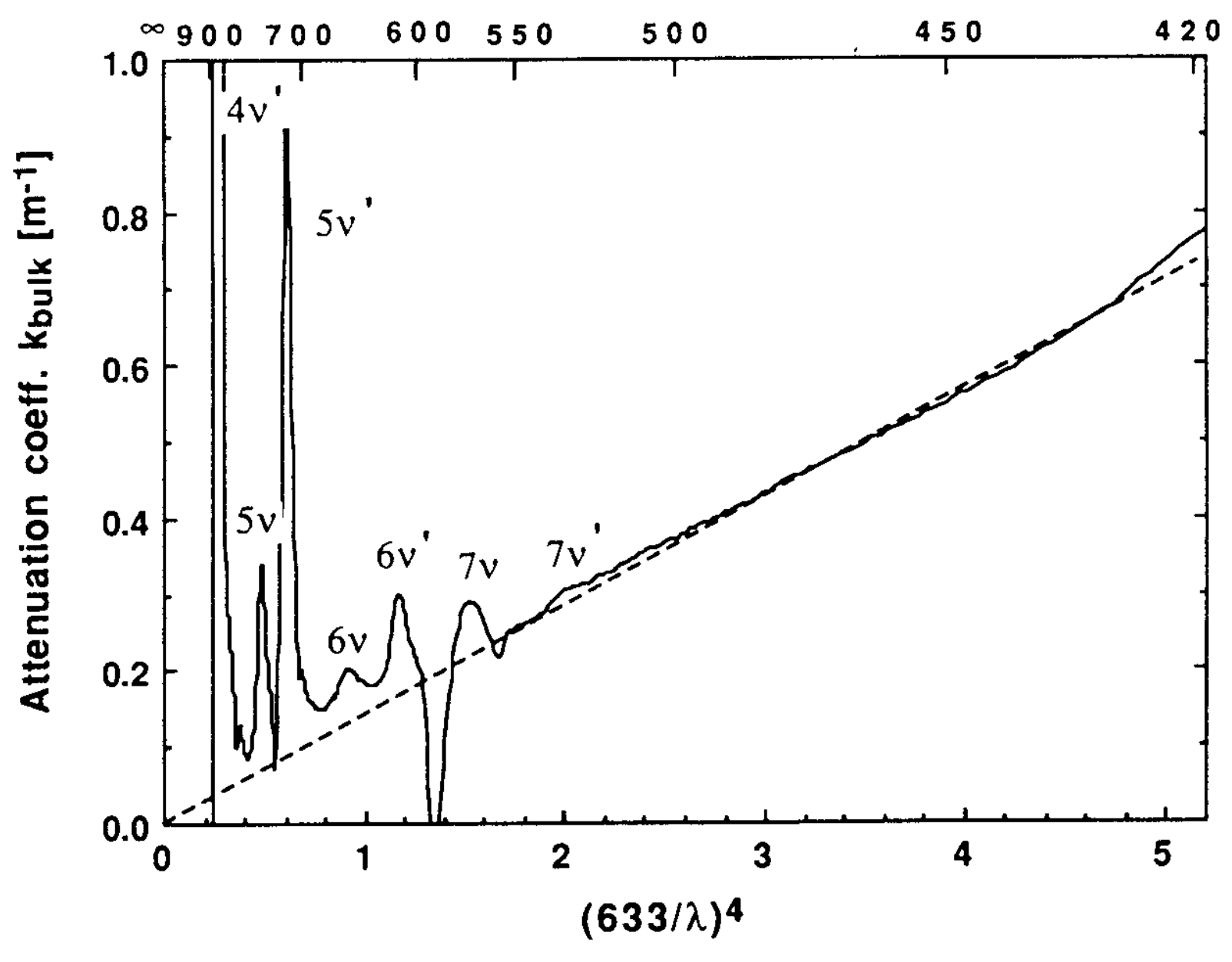

Fig. 8(b) 


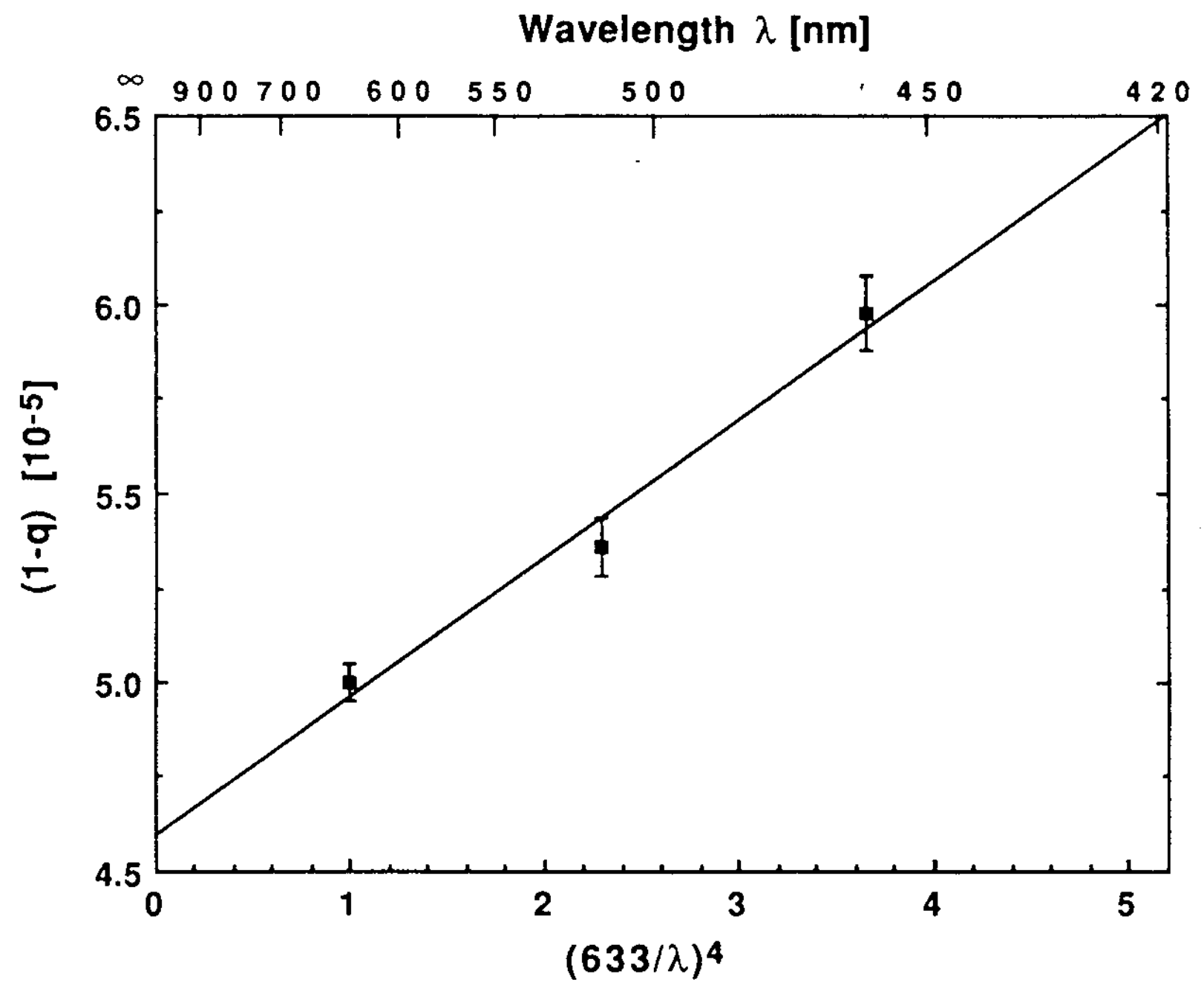

Fig. $8(c)$ 

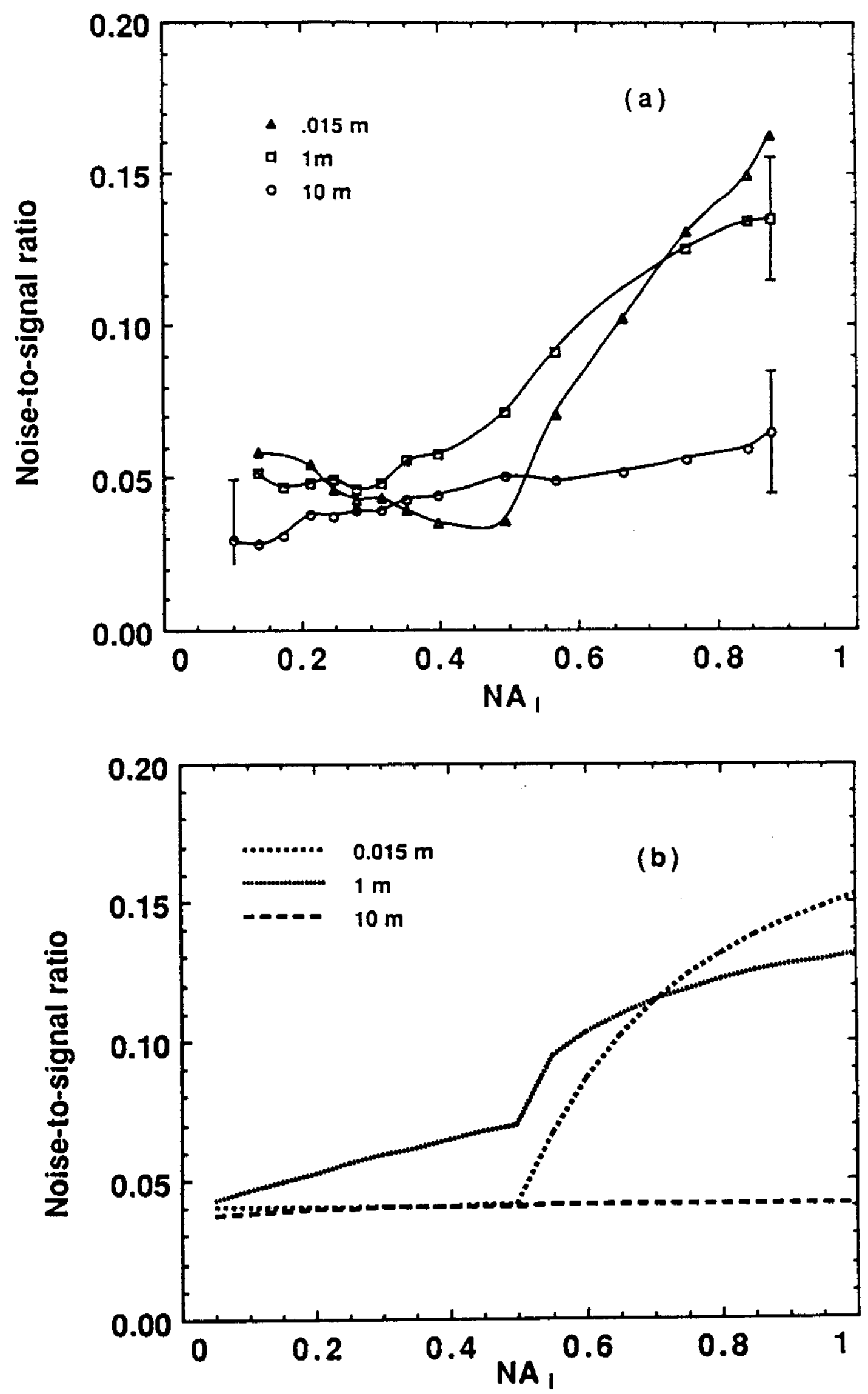

Fig. 9 